\title{
Change in C1q deposition in C1q nephropathy
}

\author{
Kazunari Kaneko • Masafumi Hasui • Ken Hatae • \\ Hidenori So • Ritsuko Katafuchi
}

Received: 22 February 2009 / Accepted: 23 February 2009 / Published online: 14 March 2009

(C) IPNA 2009

Sirs,

We read with interest the article entitled "A single-center study of C1q nephropathy in children" by Roberti et al. in the latest issue of this journal [1].

They reviewed the records of 14 children with $\mathrm{Clq}$ nephropathy $(\mathrm{C} 1 \mathrm{qN})$ and found a high incidence in pediatric patients $(14 / 264,5.3 \%)$, with variable clinical presentation. Fortunately, it is reported that $\mathrm{C} 1 \mathrm{qN}$, despite a high incidence of steroid resistance and poor renal prognosis in the previous reports, is characterized by a clinically excellent response and fair renal survival with the addition of further immunosuppression. We would like to add information regarding a change in deposition of $\mathrm{Clq}$ in parallel with clinical course.

A 1-year-old girl was referred to us from a local hospital because of heavy proteinuria and edema, with a diagnosis of idiopathic nephritic syndrome (NS). She was treated orally with prednisolone for 6 weeks, with no decrease in the amount of proteinuria. A diagnosis of steroid-resistant NS prompted us to perform renal biopsy: the histology showed a minimal change lesion on light microscopy, dominant positive staining of $\mathrm{C} 1 \mathrm{q}$ in the mesangium, with lesser extent of $\mathrm{C} 3$, immunoglobulin (Ig)A, and $\mathrm{IgM}$, on

K. Kaneko $(\bowtie) \cdot$ M. Hasui

Department of Pediatrics, Kansai Medical University,

2-3-1 Shin-machi,

Hirakata-shi, Osaka 5731191, Japan

e-mail:kanekok@hirakata.kmu.ac.jp

K. Hatae $\cdot$ H. So

Department of Pediatrics, Fukuoka Red Cross Hospital,

Fukuoka, Japan

R. Katafuchi

Department of Nephrology,

National Fukuoka-Higashi Medical Center,

Fukuoka, Japan immunofluorescence microscopy, and the presence of electron-dense deposits in the paramesangial area on electron microscopy. There were no serological or clinical findings indicating lupus nephritis, which frequently mimics $\mathrm{C} 1 \mathrm{qN}$ in renal histology. Based on these findings, the pathological diagnosis of $\mathrm{ClqN}$ was made. Cyclosporine A (CsA) was chosen to treat her steroid-resistant NS and induced the disappearance of the proteinuria in 2 weeks. Thereafter, continuous oral administration of CsA with trough level of $60-80 \mathrm{ng} / \mathrm{ml}$ maintained her remission for 2.5 years until now. The second renal biopsy, which we performed recently to investigate the CsA-induced nephropathy, revealed a minimal change lesion and a faint staining of $\mathrm{Clq}$.

There have been several reports regarding both the clinical and pathological characteristics of $\mathrm{C} 1 \mathrm{qN}$ in children [1-5]. In those studies, however, change in C1q deposition revealed by serial renal biopsy was rarely reported. From our experience, we would like to suggest that clinical improvement might alleviate or even cause the disappearance of $\mathrm{Clq}$ deposits from the mesangium in $\mathrm{ClqN}$.

\section{References}

1. Roberti I, Baqi N, Vyas S, Kim D (2009) A single-center study of C1q nephropathy in children. Pediatr Nephrol 24:77-82

2. Iskandar SS, Browning MC, Lorentz WB (1991) C1q nephropathy: a pediatric clinicopathologic study. Am J Kidney Dis 18:459-465

3. Fukuma Y, Hisano S, Segawa Y, Niimi K, Tsuru N, Kaku Y, Hatae K, Kiyoshi Y, Mitsudome A, Iwasaki H (2006) Clinicopathologic correlation of C1q nephropathy in children. Am J Kidney Dis 47:412-418

4. Kersnik Levart T, Kenda RB, Avgustin Cavić M, Ferluga D, Hvala A, Vizjak A (2005) C1Q nephropathy in children. Pediatr Nephrol 20:1756-1761

5. Lau KK, Gaber LW, Delos Santos NM, Wyatt RJ (2005) C1q nephropathy: features at presentation and outcome. Pediatr Nephrol 20:744-749 